Original Article

\title{
Reliability and validity of measuring respiration movement using a wearable strain sensor in healthy subjects
}

\author{
Haijuan Liu ${ }^{1,2)^{*}}$, Shaopeng Guo ${ }^{2)}$, Kaipei Zheng ${ }^{3)}$, Xiaojun Guo, PhD ${ }^{3)}$, \\ Tsugumi Kuramoto-Ahuja, PT, MA ${ }^{1)}$, Tamae Sato, PT, MS ${ }^{1)}$, Ko Onoda, PT, PhD ${ }^{1)}$, \\ Hitoshi Maruyama, PT, $\mathrm{PhD}^{1)}$ \\ 1) Department of Physical Therapy, International University of Health and Welfare: 2600-1 \\ Kitakanemaru, Ohtawara city, Tochigi 324-8501, Japan \\ 2) China Rehabilitation Research Center, China \\ 3) Shanghai Jiao Tong University, China
}

\begin{abstract}
Purpose] The purpose of this research was to verify the reliability and validity of measuring respiration movement using a wearable strain sensor (WSS) which has been developed newly for clinical objective assessment. [Subjects and Methods] The 21 healthy male students were advised to conduct a breathing movement using measuring tape (MT) and WSS respectively, which was the measured at four locations on chest and abdomen wall. The different degree of chest expansion from maximal end of expiration to maximal end of inspiration was confirmed at each location. The intra-rater ICC $(1,1)$ with $95 \%$ confidence interval was used to assess the test-retest reliability, Pearson's correlation analysis was performed to establish the validity. [Results] All ICC values for intrarater reliability were from 0.94 to 0.98 at all locations, which means that there is a high correlation. All values for validity showed significantly positive, indicating that there is a correlation between the measuring tape and WSS at four locations. [Conclusion] Compared to the measuring tape, WSS has been proved to have high reliability and validity. The finding of this research indicated that WSS is reliable to use for objective measurement of respiratory movements on the chest and abdomen wall in clinical assessment.

Key words: Reliability, Validity, Wearable strain sensor
\end{abstract}

(This article was submitted Apr. 17, 2017, and was accepted Jun. 5, 2017)

\section{INTRODUCTION}

Respiration movement is an important component in the assessment of pulmonary function. In clinical practice, respiration movement is generally assessed by inspection and palpation in the physical examination, which not only provides real-time observation but does not require any other special measuring devices. Nevertheless, physical examinations are not quantitative, but qualitative analysis based on the experience of a particular examiner. Measurement devices include a magnetometers ${ }^{1)}$, respiratory inductive plethysmography ${ }^{2)}$, optoelectronic plethysmography ${ }^{3-7)}$, and respiratory movement measured devices ${ }^{8,9)}$, which can be applied for quantitative measurement. Because of the high cost and the complex settings of those devices, they are not suitable for clinical practice sometimes. A scale to assess breathing movements which was only depended on the experiences of the assessor, may not have sufficient reliability ${ }^{10)}$.

Measurement of chest expansion is a simple and practical method for assessing subjects' respiratory function. Shobo et al. found that there was a highly positive correlation between chest expansion and changes in the chest volumes at all indicated locations ${ }^{11,12}$. However, measuring devices of chest expansion were both 3-dimensional motion analyzer and a spherical reflec-

*Corresponding author. Haijuan Liu (E-mail: liuhaijuan0813@sina.com)

(C2017 The Society of Physical Therapy Science. Published by IPEC Inc.

(c) (1) $\odot$ This is an open-access article distributed under the terms of the Creative Commons Attribution Non-Commercial No Derivatives

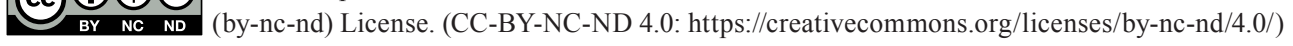


Table 1. The characteristics of all male subjects in this study $(n=21)$

\begin{tabular}{lcccc}
\hline & \multicolumn{4}{c}{ Mean \pm SD } \\
\hline Age $(\mathrm{yrs})$ & \multicolumn{4}{c}{$24.0 \pm 2.7$} \\
Height $(\mathrm{cm})$ & $173.8 \pm 3.9$ & \\
Weight $(\mathrm{kg})$ & \multicolumn{4}{c}{$67.6 \pm 7.5$} \\
BMI $\left(\mathrm{kg} / \mathrm{m}^{2}\right)$ & \multicolumn{4}{c}{$22.4 \pm 2.6$} \\
\hline IC $(\mathrm{cm})$ & Axilla & Xiphoid process & 10 th rib & Umbilicus \\
\cline { 2 - 5 } & $93.2 \pm 5.5$ & $83.2 \pm 6.0$ & $78.4 \pm 7.2$ & $80.3 \pm 8.3$ \\
\hline
\end{tabular}

All values were shown as mean $\pm \mathrm{SD}$ (standard deviation).

BMI: body mass index; IC: initial circumference (average of during ordinary respiration after measuring three time)

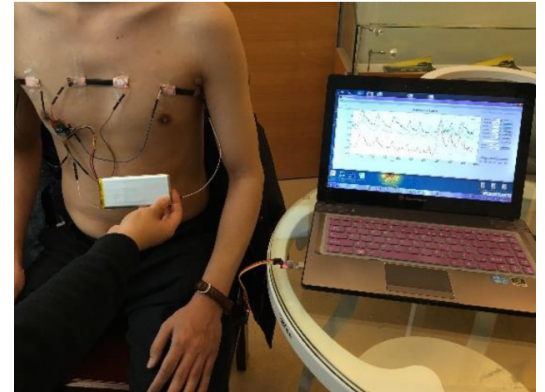

Fig. 1. Respiration measurement using wearable strain sensor (WSS)

tive marker, which were required to conduct in a special experimental environment. It is inconvenient for patients in a clinical setting. Especially, it was important to illustrate that there may be considerable sliding of the skin over the rib cage during deep breathing, this was called "skin move artifact"13,14). Thus, it causes measurement errors. The difference of chest expansion between the maximum exhalation and maximum inspiration measured chest circumferences at axilla, xiphoid process and 10th rib was correlated with respiratory function ${ }^{15}$. The procedure of measurement would take a longer duration, which may increase the fatigue of patients. Therefore, a wearable strain sensor (WSS) was developed for easier to use and more objective measurement of chest expansion in a clinical setting. The purpose of this research is to verify the reliability and validity of measuring respiration movement using a wearable strain sensor (WSS) which has been developed newly for clinical objective assessment.

\section{SUBJECTS AND METHODS}

The study was conducted at Shanghai Jiao Tong University. Twenty-one healthy male students were recruited from the university in this research, the characteristics of whom were shown in Table 1, and those with a history of respiratory, circulatory, or neurological disorders were excluded. The study was approved by the Ethics Committees of International University of Health and Welfare (No.16-Io-176 and 177) and China Rehabilitation Research Center (CRRC-IEC-RF-SC-005-01). All the subjects signed their informed consent.

The initial circumferences of chest (axilla, xiphoid process and 10th rib) ${ }^{13)}$ and abdomen (umbilicus) ${ }^{11)}$ were measured by a measuring tape (length, $100 \mathrm{~cm}$. made in Guangzhou Fengren Ltd., China) during ordinary respiration and the axilla location was the biggest circumference in all the four locations. The measurement with a wearable strain sensor comprises three sensors connected to each other in parallel. They were stuck to the chest and abdomen wall with medical adhesive tape to monitor the movement and the expansion of the thoracoabominal skin. The output of the sensors is connected to a selfdesigned circuit board, which is also stuck onto the chest or abdomen wall, as illustrated in Fig 1. The circuit board received the sensor signals, and communicated wirelessly with a computer through Bluetooth ${ }^{16,17)}$. The monitored data are processed using software (MATLAB Version 7.11.0.584 (R2010b), License Number: 161051), and the workable strain ranges of sensors were calculated using a spreadsheet software (Microsoft Excel, USA).

In the preliminary measurement, the stretched length of wearable strain sensor was selected using $5 \%$ of axilla circumference $(5 \% \mathrm{AC})$, which was convenient and effective for measuring breathing movement. The measurement points were placed along the midline of chest and abdomen through horizontal midpoint of circumference at four locations such as the axilla, xiphoid process, 10 th rib and umbilicus. Besides, they were also placed along the second 5\% AC bilaterally from the midaxillary line to midline of chest and abdomen at the axilla, xiphoid process, 10th rib and umbilicus, respectively, to escape the cover for the limbs. There were three measurement points for each location, which were matched with the three strain sensors connected to each other in parallel. This means that the four locations had totally twelve points.

The 21 subjects were advised to conduct a breathing movement with their shirt off and pants loosed in a standing position. Initial circumference was measured at each location as a starting point, and then from the maximal end of expiration to the maximal end of inspiration respectively, confirming the different degrees of chest expansion between the two. Every subject conducted the breathing movement for three times and one average value was needed. Then, every subject wearing three strain sensors at each measurement point was requested to conduct the same breathing movement. While each subject was breathing for three times at each location, simultaneously the data from the three parallel sensors were collected. After the three different values were acquired from each sensor, their average value was calculated. There was a break between the first and second breathing movements, allowing each subject to feel fine before the second trial.

When one rater measured with the use of a measuring tape and WSS with the synchoronal instructional procedure of respiratory movement, the required criteria of the oral instruction in the two approaches was exactly same. The subject's test was arranged in an hour after the meal in order to keep fasting state, the retest was conducted at the same time on the second 
Table 2. The test-retest raw data (Mean \pm SD) of respiratory measurement using WSS

\begin{tabular}{|c|c|c|c|c|c|c|}
\hline & \multicolumn{2}{|c|}{ Left } & \multicolumn{2}{|c|}{ Middle } & \multicolumn{2}{|c|}{ Right } \\
\hline & Test $(\mathrm{cm})$ & Retest $(\mathrm{cm})$ & Test $(\mathrm{cm})$ & Retest $(\mathrm{cm})$ & Test $(\mathrm{cm})$ & Retest $(\mathrm{cm})$ \\
\hline Axilla & $0.44 \pm 0.28$ & $0.43 \pm 0.29$ & $0.35 \pm 0.21$ & $0.36 \pm 0.20$ & $0.45 \pm 0.22$ & $0.44 \pm 0.22$ \\
\hline Xiphoid process & $0.33 \pm 0.21$ & $0.36 \pm 0.19$ & $0.34 \pm 0.20$ & $0.34 \pm 0.19$ & $0.35 \pm 0.20$ & $0.37 \pm 0.20$ \\
\hline 10th rib & $0.60 \pm 0.26$ & $0.61 \pm 0.24$ & $0.57 \pm 0.22$ & $0.58 \pm 0.22$ & $0.53 \pm 0.22$ & $0.55 \pm 0.21$ \\
\hline Umbilicus & $0.45 \pm 0.17$ & $0.44 \pm 0.16$ & $0.46 \pm 0.24$ & $0.47 \pm 0.25$ & $0.45 \pm 0.24$ & $0.45 \pm 0.25$ \\
\hline
\end{tabular}

WSS: wearable strain sensor

Table 3. The test-retest reliability of respiratory measurement using WSS for ICC $(1,1)$

\begin{tabular}{lccc}
\hline & Left & Middle & Right \\
\hline Axilla & 0.97 & 0.97 & 0.98 \\
Xiphoid process & 0.94 & 0.98 & 0.95 \\
10th rib & 0.97 & 0.98 & 0.96 \\
Umbilicus & 0.96 & 0.98 & 0.98 \\
\hline
\end{tabular}

WSS: wearable strain sensor $\mathrm{p}<0.05,95 \%$ confidence interval. ICC: intraclass correlation coefficient

Table 4. The raw data (Mean \pm SD) of the MT compared with WSS for measuring respiratory movement

\begin{tabular}{|c|c|c|c|c|}
\hline & \multicolumn{2}{|c|}{ Left } & \multirow{2}{*}{$\begin{array}{c}\text { Middle } \\
\text { WSS (cm) }\end{array}$} & \multirow{2}{*}{$\begin{array}{c}\text { Right } \\
\text { WSS (cm) }\end{array}$} \\
\hline & MT (cm) & WS (cm) & & \\
\hline Axilla & $5.43 \pm 1.56$ & $0.35 \pm 0.31$ & $0.36 \pm 0.29$ & $0.36 \pm 0.27$ \\
\hline Xiphoid process & $5.19 \pm 1.92$ & $0.41 \pm 0.21$ & $0.42 \pm 0.20$ & $0.42 \pm 0.20$ \\
\hline 10th rib & $6.71 \pm 1.86$ & $0.56 \pm 0.22$ & $0.60 \pm 0.19$ & $0.56 \pm 0.21$ \\
\hline Umbilicus & $4.04 \pm 1.6$ & $0.27 \pm 0.19$ & $0.29 \pm 0.21$ & $0.27 \pm 0.21$ \\
\hline
\end{tabular}

MT: measuring tape; WSS: wearable strain sensor

Table 5. The validity of the WSS for measuring respiratory movement

\begin{tabular}{llll}
\hline & Left & Middle & Right \\
\hline Axilla & $0.72^{* *}$ & $0.73^{* *}$ & $0.73^{* *}$ \\
Xiphoid process & $0.80^{* *}$ & $0.82^{* *}$ & $0.81^{* *}$ \\
10th rib & $0.83^{* *}$ & $0.85^{* *}$ & $0.82^{* *}$ \\
Umbilicus & $0.79^{* *}$ & $0.82^{* *}$ & $0.79^{* *}$ \\
\hline WSS: wearable strain sensor & \\
${ }^{*} \mathrm{p}<0.05,{ }^{* *} \mathrm{p}<0.01$ was significant correlation at 0.01 level (bilateral).
\end{tabular}

day of test. There were no learning effects, furthermore every test was controlled in the same experimental condition.

The demographics of all subjects were shown as mean \pm SD (standard deviation). The intra-rater ICC $(1,1)$ with $95 \%$ confidence interval was used to assess for test-retest reliability and Pearson correlation analysis was performed to establish the related validity of respiratory measurement using the WSS. All data were calculated and analyzed using the IBM SPSS Statistics version 19.0 for Windows software as well as values of less than 0.05 were statistically significant level.

\section{RESULTS}

The test-retest raw data of respiratory measurement using WSS were shown in Table 2, and the intra-rater ICC $(1,1)$ values for test-retest reliability were presented in Table 3. All ICC values for intra-rater reliability were from 0.94 to 0.98 at all locations, there is a high correlation based on the standard of Landis et al ${ }^{18)}$.

The raw data of the MT compared with WSS for measuring respiratory movement were indicated in Table 4 as well as to determine the validity of the WSS for measuring respiratory movement, Pearson correlation coefficient was calculated in Table 5. All the values for validity had significant positive correlations on respiratory movement between measuring tape and WSS at four locations $(\mathrm{p}<0.01)$. 


\section{DISCUSSION}

This study confirmed the reliability and validity of the innovatively developed wearable strain sensor. The motivation of developing WSS was due to both the high subjectivity existing and complex limited setting during respiratory movement assessment in clinical practice at present. The reliability of WSS with a high correlation and validity of WSS with relatively high values, compared to the results of the measuring tape, were valuable. These findings indicate that the WSS is available as a convenient approach for objective measurement of respiratory movements on the chest and abdomen wall in clinical assessment.

In preliminary measurement, the stretching length of wearable strain sensor with $5 \%$ of axilla circumference $(5 \% \mathrm{AC})$ was selected which is convenient and effective for measuring respiratory movement. This length was assumed based on the following reasons:1) Taking the same proportion of their individual AC for each subject, and performing the same measuring procedure, the results obtained from the test are comparable. 2) 5\% AC was considered based on axilla circumference mean $\pm \mathrm{SD}$ of the subject's sample, which is the longest on the part of both length and coverage compared to $5 \% \mathrm{AC}$ of the other three locations. Moreover, it's convenient for calculation and more effective for measuring expansion of chest and abdomen wall. 3) There was one developed size $(3.5 \mathrm{~cm})$ based on initial axilla circumference $(93.2 \pm 5.46)$ for the original length of strain sensor with the loading of strain in the range of $0-250 \%{ }^{14)}$. Owing to the length with $3.5 \mathrm{~cm}$ of three-channels, WSS was placed matching to $5 \% \mathrm{AC}$ during ordinary breathing meanwhile the original length with $3.5 \mathrm{~cm}$ was stretched to the individual $5 \% \mathrm{AC}$ of each subject as a starting point to measure. Therefore, the original length of WSS with $3.5 \mathrm{~cm}$ was applied in tquiehis study. In fact, the original length of WSS was not correlated with the percentage of circumference of chest and abdomen wall for measuring respiratory movement. The reliability and validity of WSS with $3.5 \mathrm{~cm}$ were more essential in clinical evidence.

In this study, the reliability ICC $(1,1)$ of WSS at each location performed high values. The values of both left and right of chest and abdomen wall, however were a little lower compared with middle place. The analyzed reason was the muscles strength with unbalance on the trunk bilateral or trunk with side-flexion in standing position during respiration movement. Although the same breathing was conducted, the measured stretching skin of bilateral trunk was diverse by WSS with high sensitivity and high stretchability based on stretching mechanism ${ }^{14)}$.

Regarding the validity, all the values of this study presented a significant correlation $(\mathrm{p}<0.01)$ between WSS and measuring tape. Therefore, we concluded that the validity of measuring respiration movement using WSS was satisfactory. The measuring tape was a reference variable in this study which was a circumference test of chest and abdomen wall that provided a difference between maximal end of expiration and, maximal end of inspiration. This test has limitations as follows: because there are four locations which were needed to measure on chest and abdomen wall, measuring respiratory movement for three times at each location, totally taking approximately 25 minutes, the whole measuring procedure for each subject may cause fatigue even for young people; the test may be not suitable for subjects with both same circumference size on chest and abdomen wall and similar respiratory movement, because of the measured results among the subjects without any distinction using measuring tape. The WSS with high sensitivity, however is more objective accuracy, which can capture signals with the tiny muscle movement caused by blinking ${ }^{14)}$; As noted above, the validity values of WSS were not significantly high from 0.72 to 0.85 . The validity of axilla location were the lowest values compared with another three locations, the cause of which may be that the measuring tape was caught by both upper limbs in the armpit, resulting in reading errors. The validity values of both left and right of chest and abdomen wall were a little lower compared with middle place as well, followed by that middle place on the chest and abdomen wall will be more reliable and valid within three places for measuring respiratory movement.

In clinical application, an objective measurement of respiratory movement may facilitate a more meaningful assessment of respiratory conditions which can be shared among professionals with existing respiratory problems. It is considered that WSS may replace the physical examination as an objective measurement for respiratory movement in clinical practice, because this study presented that WSS has been proved to have a high degree of reliability and validity, and without limitation of posture or environment during respiration measurement.

Nevertheless, there are some limitations in this study: the communicated wirelessly computer through Bluetooth would be inconvenient compared with a smartphone; and the measurement of ordinary respiration cycles would be needed in patients with respiratory problems. Although the WSS may be useful as assisting assessment device for respiratory movement to augment objective measurement, further studies of respiratory measurement using WSS would be more valuable in patents with respiration problems.

\section{Conflicts of interest}

There are no conflicts of interest. 


\section{REFERENCES}

1) Sharp JT, Goldberg NB, Druz WS, et al.: Relative contributions of rib cage and abdomen to breathing in normal subjects. J Appl Physiol, 1975, 39: 608-618. [Medline]

2) Gilbert R, Auchincloss JH Jr, Peppi D: Relationship of rib cage and abdomen motion to diaphragm function during quiet breathing. Chest, 1981, 80: 607-612. [Medline] [CrossRef]

3) Cala SJ, Kenyon CM, Ferrigno G, et al.: Chest wall and lung volume estimation by optical reflectance motion analysis. J Appl Physiol 1985, 1996, 81: 26802689. [Medline]

4) Aliverti A, Dellacá R, Pelosi P, et al.: Optoelectronic plethysmography in intensive care patients. Am J Respir Crit Care Med, 2000, 161: 1546-1552. [Medline] [CrossRef]

5) Aliverti A, Dellacà R, Pelosi P, et al.: Compartmental analysis of breathing in the supine and prone positions by optoelectronic plethysmography. Ann Biomed Eng, 2001, 29: 60-70. [Medline] [CrossRef]

6) Binazzi B, Lanini B, Bianchi R, et al.: Breathing pattern and kinematics in normal subjects during speech, singing and loud whispering. Acta Physiol (Oxf), 2006, 186: 233-246. [Medline] [CrossRef]

7) Romei M, Mauro AL, D'Angelo MG, et al.: Effects of gender and posture on thoraco-abdominal kinematics during quiet breathing in healthy adults. Respir Physiol Neurobiol, 2010, 172: 184-191. [Medline] [CrossRef]

8) Ragnarsdóttir M, Kristinsdóttir EK: Breathing movements and breathing patterns among healthy men and women 20-69 years of age. Reference values. Respiration, 2006, 73: 48-54. [Medline] [CrossRef]

9) Kaneko H, Horie J: Breathing movements of the chest and abdominal wall in healthy subjects. Respir Care, 2012, 57: 1442-1451. [Medline] [CrossRef]

10) Kaneko H: Assessing the reliability and validity of a newly developed breathing movement measuring device. J Phys Ther Sci, 2013, 25: 425-429. [CrossRef]

11) Shobo A, Kakizaki K: Relationship between chest expansion and the change in chest volume. Rignku ryohougaku, 2014, 29: 881-884 (in Japanese).

12) Shobo A, Kakizaki K: Effects of two sitting positions on chest volume. Rignku ryohougaku, 2015, 30: 499-502 (in Japanese).

13) Pedotti A, Ferrigno G: Opto-electronics based systems. In: Three-Dimensional Analysis of Human Movement, Allard P, Stokes LAF, Bianchi JP (eds.), Champaign: Human Kinetics, 1995, 4, pp 57-78.

14) Morgan MD, Gourlay AR, Silver JR, et al.: Contribution of the rib cage to breathing in tetraplegia. Thorax, 1985, 40: 613-617. [Medline] [CrossRef]

15) Tahira K, Sukizaki T, Senjyu H, et al.: Investigation of correlation between standard values of chest expansion difference and pulmonary function in middleaged and older adults. Rigaku ryohougaku, 1993, 20 (suppl): 84 (in Japanese).

16) Wang C, Li X, Gao E, et al.: Carbonized silk fabric for ultrastretchable, highly sensitive, and wearable strain sensors. Adv Mater, 2016, 28: 6640-6648. [Medline] [CrossRef]

17) Wei H, Chen S, Zhou B, et al.: Highly sensitive and transparent strain sensor based on thin elastomer film. Electron Device Lett, 2016, 37: 667-670.

18) Landis JR, Koch GG: The measurement of observer agreement for categorical data. Biometrics, 1977, 33: 159-174. [Medline] [CrossRef] 